\title{
What is light?
}

\section{Students' reflections on the wave-particle duality of light and the nature of physics}

\author{
Ellen Karoline Henriksen ${ }^{1}$, Carl Angell ${ }^{1}$, Arnt Inge Vistnes ${ }^{1}$ \& Berit Bungum ${ }^{2}$
}

1 Department of Physics, University of Oslo

2 Department of Teacher Education, Norwegian University of Science and Technology, Trondheim

\begin{abstract}
Quantum physics describes light as having both particle and wave properties; however, there is no consensus about how to interpret this duality on an ontological level. This article explores how preuniversity physics students, while working with learning material focusing on historical-philosophical aspects of quantum physics, interpreted the wave-particle duality of light and which views they expressed on the nature of physics. A thematic analysis was performed on 133 written responses about the nature of light, given in the beginning of the teaching sequence, and 55 audio-recorded small-group discussions addressing the wave-particle duality, given later in the sequence. Most students initially expressed a wave and particle view of light, but some of these gave an "uncritical duality description", accepting without question the two ontologically different descriptions of light. In the small-group discussions, students expressed more nuanced views. Many tried to reconcile the two descriptions using semi-classical reasoning; others entered into philosophical discussions about the status of the current scientific description of light and expected science to come up with a better model. Some found the wave description of light particularly challenging and lacked a conception of "what is waving". Many seemed to implicitly take a realist view on the description of physical phenomena, contrary to the Copenhagen interpretation which is prevalent in textbooks. Results are discussed in light of different interpretations of quantum physics, and we conclude by arguing for a historical-philosophical perspective as an entry point for upper secondary physics students to explore the development and interpretation of quantum physical concepts.
\end{abstract}

Keywords: wave-particle duality; interpretation of quantum physics; nature of physics; upper secondary physics; nature of light

\section{Introduction}

Light is a fundamental phenomenon in physics, and the question "What is light?" has been asked by humans for centuries. In the early $20^{\text {th }}$ century, quantum mechanics was developed and was soon successful in describing and predicting experimental results for quantum-scale phenomena by means of mathematics. Today it forms part of the theoretical foundation for development of new technologies such as quantum computers. This theoretical foundation includes a description of light as having both particle properties and wave properties, referred to as the wave-particle duality. However, there is no 
consensus about how to interpret quantum physics, including the contemporary scientific description of light, on an ontological level (Bunge, 2003).

Physics students, from secondary school and until they encounter quantum mechanics in university, are presented with the wave-particle duality of light. However, the wave and particle descriptions are contradictory in a classical sense. Students may find it hard to reconcile two descriptions of the same phenomenon that, despite the great predictive and descriptive power of each, are so different on the conceptual and ontological level (Hubber, 2006). Ontological questions relate to considerations about what light is; in what sense can we say that the waves and particles described by quantum physics exist? However, such philosophical questions are seldom discussed in physics education. Greca and Freire Jr (2014a) have argued that this may be because the very existence of several interpretations of quantum theory seems to be an inconvenient truth for the teaching of physics.

In a review of research literature on how quantum concepts should be introduced in physics teaching, Greca and Freire Jr (2014b) found that science education researchers give different answers and suggest different approaches. However, they also noted that the current research base does not provide any strong evidence for advocating one way over another, because few of the proposals have been tested. This paper contributes to the field by presenting empirical results concerning how preuniversity physics students, while working with specific learning material, interpret the wave-particle duality of light and how they look upon the nature of physics. The results are discussed in light of perspectives on the ontology and epistemology of quantum physics. Finally, we discuss how, and to what extent, it is feasible to introduce philosophical perspectives on the scientific description of light in upper secondary school physics.

\subsection{The nature of light: A brief historical overview}

Until around 1800, most scientists thought that light consisted of streams of particles, in line with the view expressed by Newton in "Opticks" (Newton, 1952). However, contemporaries of Newton, like Robert Hooke and Christian Huygens, conceived of light as waves. In 1803 Thomas Young performed his famous double-slit experiment, which supported the wave model (Young, 1804). But even Young's experiment did not convince most physicists at the time.

A major change took place about 15 years later. In 1818 the French Academy of Sciences announced a competition to explain the properties of light. August-Jean Fresnel participated and launched a wave theory for light. The famous mathematician Siméon Poisson was a member of the evaluation committee. He supported the particle model of light, and tried to show that Fresnel's theory was wrong. Poisson predicted that if light had a wave nature, one should expect to find a bright spot in the middle of the shadow of a circular disk. Poisson expected that such behaviour would be impossible. However, the experimental physicist Jean Arago decided to carry out the experiment, and found in fact the bright spot exactly as predicted. The spot is now called the Arago spot or even the Poisson spot (see e.g. Kragh \& Pedersen, 1992).This experiment is often seen as an experimentum crucis that greatly changed scientific opinion on the nature of light in favour of the wave model. Fifty years later, Maxwell's equations described light as a part of the spectrum of electromagnetic waves, further supporting the wave theory for light.

Max Planck introduced the notion of energy quanta in his derivation of the law of blackbody 
radiation in 1900 by assuming that the oscillation energy of a molecule in the wall of a container could only have certain values. At that time he regarded the energy quanta merely as a calculating device, and only related to absorption and emission (Planck, 1900). It was Albert Einstein who, in 1905, described the photoelectric effect by suggesting that light consists of indivisible energy quanta, indicating that Maxwell's wave theory had limited validity:

It seems to me that the observations associated with blackbody radiation, fluorescence, the production of cathode rays by ultraviolet light, and other related phenomena connected with the emission or transformation of light are more readily understood if one assumes that the energy of light is discontinuously distributed in space. In accordance with the assumption to be considered here, the energy of a light ray spreading out from a point source is not continuously distributed over an increasing space but consists of a finite number of energy quanta which are localized at points in space, which move without dividing, and which can only be produced and absorbed as complete units (Arons \& Peppard, 1965, p. 368)

The light quanta, later to be known as photons or "light particles", played a crucial role in explaining the photoelectric effect, and the model has great explanatory power for many phenomena. Thus, light, which according to Maxwell had been considered as a form of electromagnetic waves, must also be thought of as particle-like, localized packets of energy. This dual description of light is known as waveparticle duality. The origin of this concept is to be found in a paper by Einstein from 1909 (see Gingras, 2015):

It is even undeniable that there is an extensive group of facts concerning radiation that shows that light possesses certain fundamental properties that can be understood far more readily from the standpoint of Newton's emission theory of light than from the standpoint of the wave theory. It is therefore my opinion that the next stage in the development of theoretical physics will bring us a theory of light that can be understood as a kind of fusion of the wave and emission theories of light (Einstein, 1989, p. 379)

Niels Bohr regarded the wave-particle duality as a fundamental property of nature. He saw such duality as one aspect of the concept of complementarity, which he for the first time introduced in a lecture in 1927 in Como, Italy: "The two views of the nature of light are rather to be considered as different attempts at an interpretation of experimental evidence in which the limitation of the classical concepts is expressed in complementary ways" (Bohr, 1928, p. 581). Bohr also emphasized that we are not dealing with contradictory but with complementary pictures of the phenomena (see e.g. Held, 1994).

Today, it is widely accepted in the physics community that light and other electromagnetic radiation exhibit wave-particle duality. Light behaves like waves in some experiments, and like particles in other experiments. Interference and diffraction demonstrate wave behaviour, while emission and absorption demonstrate the particle behaviour. What the duality means on an ontological level is, however, still ambiguous and a field of discussion, which will be briefly reviewed in the next section.

\subsection{Philosophical considerations: Realism versus the Copenhagen interpretation in physics and physics education}

Ontology is essential for how the wave-particle duality is interpreted and has consequences for how quantum physics is presented in physics education, and Baily and Finkelstein (2014) pointed out how students' underlying ontological conceptions are related to how they conceptualize light. 
One philosophical position concerning ontological questions in quantum physics is that it is not the concern of physics to reveal the "true" nature of phenomena; physics can only provide us with calculation tools that can be used to predict the outcome of experiments or processes we can observe. This instrumentalist approach is inherent in the so-called Copenhagen interpretation of quantum physics. Yet the Copenhagen Interpretation is not a monolithic doctrine but rather denotes a family of interpretations (Camilleri, 2009; Cordero, 2003). The core of these viewpoints is that a physical system does not have a definite physical state prior to being measured and that quantum mechanics can only predict the probabilities that measurements will produce certain results. For light, wave and particle models are seen in the Copenhagen interpretation as two complementary descriptions where the question about the "true nature" of light is considered meaningless.

A different philosophical stance, realism, assumes that "the universe exists independently of the knowing subject or observer (...) and that a subject may get to know the universe objectively" (Bunge, 2012). Karakostas and Hadzidaki (2005, p. 607) arguing from a realist perspective, held that "the objects of science (...) reflect objective structural aspects of the physical world". This philosophical position seems to be readily applicable, for instance, to the wave model of light described by Maxwell's electrodynamics, where electric and magnetic fields are assumed to actually exist; they can be measured, and it is quite easy to imagine that a space-time point where the electric field is non-zero has a different property from space-time points where the electric field is zero.

The wave and particle notions of light are quite distinct with respect to the physical mechanisms for the propagation of light. In the double-slit experiment, a wave model is needed to account for the interference pattern formed on a screen by light after passing through a double slit. However, the interference pattern is made up of individual impacts on the detection screen. The common interpretation is that each impact on the detector is due to absorption of one discrete photon. This interpretation, however, reveals a key problem, since the notion of individual photon impacts implicitly assumes a realist description. In the Copenhagen description, the combination of a wave and particle approach is not really a problem as long as physics accurately predicts the outcome of the experiment. Within a realist understanding, on the other hand, a particle is most often understood as something like a tiny golf ball. It is an entity that is distinct from the surroundings, it is indivisible, and it remains the very same particle throughout its journey, from it is created until it is destroyed. A realist view makes it problematic to think of light as a real wave for some part of its movement through space and time, and as a real particle at a later stage. The difference in physical mechanisms behind wave propagation and a particle trajectory will forbid such a transition within a realist description.

Lautesse, Valls, Ferlin, Héraud, and Chabot (2015) claimed that some of the ontological challenges in quantum physics go back to Bohr's insisting on using classical concepts such as "wave" and "particle" to describe quantum physical objects. They remarked (Lautesse et al., 2015, p. 941):

...it was possible to move ahead while conserving classical concepts, with Bohr in particular theorizing this strategy. Nevertheless, some physicists have complained about the uncomfortable intellectual position imposed by this kind of conceptual conservatism.

Bohr was influenced by Ernst Mach's philosophy, the forerunner for the direction called logical positivism. Bohr (1961, p. 18) stated: 
In our description of nature the purpose is not to disclose the real essence of the phenomena but only to track down, so far as it is possible, relations between the manifold aspects of our experience.

This is an expression of the Copenhagen interpretation of quantum physics, in the sense that neither the electromagnetic wave nor the particle description should be considered as a realist description of nature. Electromagnetism is a useful theory that correctly predicts relations between the manifold aspects of our experience within the classical regime, and quantum physics, including the particle description of light, correctly predicts relations between the manifold aspects of our experience within the quantum regime. What light is, is not the concern of physics according to this tradition. As Baily and Finkelstein (2010b, p. 2) remarked:

The positivistic aspect of the Copenhagen Interpretation (the refusal to discuss meaning or reality) is arguably one reason why this particular interpretation has maintained such popularity over the years, in that it allows practicing physicists to apply quantum theory without having to worry about what is "really going on" (otherwise known as "Shut Up and Calculate!")

In many daily experiences, our thoughts and reasoning are based on realism. We think that it is a real moon we see on the evening sky, we think it is a real golf ball that moves through space from it being hit by the golf club until it hits the ground, even if we cannot see it in between. Why should it be different when we talk about photons? Isn't a photon real in the same way as a golf ball?

In order to tackle these questions, Bunge (2003) suggested that the Copenhagen interpretation of quantum physics should be replaced with a realist interpretation, and introduced the concept of "quantons" (see also Cini, 2003; Lévy-Leblond, 2003). By this he wanted to abolish the idea of waveparticle duality, and to abandon analogies with classical physics. The ambition was, by using the quanton concept, to build an understanding of light and other "quantum objects" that was totally free of the concepts of particles and waves. What is special about quantons (as opposed to "classons"), according to Bunge, is not their quantization but rather that that their basic laws are probabilistic and some of their properties, such as position and energy, are blunt (spread out) rather than sharp. Hence the quanton is supposed to be a real "quantum object" with the properties of being discrete (indivisible) but extended in both space and time determined by the Heisenberg uncertainty relation. The quanton concept was conceived to offer a true realist description of light, avoiding the issues with the ontological basis of the Copenhagen school. Cini (2003) has suggested, also from a realist position, that rather than describing quantons as blunt rather than sharp, they should be described as context-dependent. This means that "their physical properties have generally blunt values and that only occasionally a given property (e.g. position or velocity) may acquire a sharp value when the quanton interacts with a suitable piece of matter" (Cini, 2003, p. 539). Context dependence is not connected to a measurement, and hence not to any human influence, or creation, of reality. This distinguishes context dependence from complementarity associated with the Copenhagen interpretation of quantum physics stating that quantum objects have complementary properties which cannot be observed or measured simultaneously.

The mathematical formalism of quantum mechanics constitutes a body of knowledge that most physicists agree on, but the link between this formalism and a physical reality remains far from consensual (Cheong \& Song, 2014). Despite the many successes of quantum physics, our interpretation of it is still under discussion a century after its start (see e.g. Baily \& Finkelstein, 2010b; Greca \& Freire Jr, 
2014a). This does not mean that physicists generally take part in these discussions. The opposite is rather the case; many physicists take an instrumental approach where philosophical questions are not considered since the calculational rules give results that are considered correct beyond all reasonable doubt. This will inevitably also influence how one approaches the teaching of quantum mechanics to students, and lead to approaches described by Baily and Finkelstein (2010b, p. 2) this way: "the student will have to learn to work with operators and vectors in addition to functions and differential equations". With instrumentalist approaches, the challenges students and physicists encounter are seen as primarily technical. Also physics textbooks are often in line with an instrumentalist view in the tradition after Niels Bohr, which Greca and Freire Jr (2014a, p. 194) described as having "the epistemological premise that theories in physics are just devices for expressing regularities among observations".

How physicists interpret ontological aspects of the wave-particle duality of light is documented by McKagan, Perkins, and Wieman (2010), who described the design and validation of the Quantum Mechanics Conceptual Survey. This survey was intended for undergraduate students, but during the validation process the authors interviewed physics professors and found, for a question about the waveparticle duality, disagreements about which alternative is correct in the following problem:

You shoot a beam of photons through a pair of slits at a screen. The beam is so weak that the photons arrive at the screen one at a time, but eventually they build up an interference pattern as shown in the picture (...). What can you say about which slit any particular photon went through?

A. Each photon went through either the left slit or the right slit. If we had a good enough detector, we could determine which one without changing the interference pattern.

B. Each photon went through either the left slit or the right slit, but it is fundamentally impossible to determine which one.

C. Each photon went through both slits. If we had a good enough detector, we could measure a photon in both places at once.

D. Each photon went through both slits. If we had a good enough detector, we could measure a photon going through one slit or the other, but this would destroy the interference pattern.

E. It is impossible to determine whether the photon went through one slit or both.

McKagan et al. (2010) wrote that they believed that alternative $D$ is correct, and that the majority of physics professors agreed. However, they also reported that some professors preferred alternative $B$ (and even E) as the correct answer, and the researchers had difficulties finding faculty consensus on any version of this question. In other words, they did not find consensus on how to interpret the waveparticle duality.

We find the above problem from the survey interesting for two additional reasons. First, all the alternatives are based on a realist philosophical stance. The Copenhagen philosophy, which would dismiss as meaningless the question of which slit the photon passes through, is not represented at all. Second, it is remarkable that although the problem is about wave-particle duality, all the alternatives in this problem only consider light as a stream of photons. The results, as well as the anticipations underpinning the survey itself, illustrate the lack of consensus in how quantum physics should be interpreted, and how many physicists, not only physics students, tend to (perhaps implicitly) hold a realist view of quantum phenomena. 
We have in this presentation not attempted to cover the large number of alternative interpretations of quantum physics during the later years such as the "pilot wave interpretation" (see e.g. Vervoort \& Gingras, 2015), since the focus of our investigation is how students ontologically make sense of the descriptions of light as particles and as waves.

\subsection{The nature of light and the nature of science in upper secondary school physics} Presenting quantum physics to upper secondary school students involves several challenges. The success of quantum physics is the use of its mathematical formalism, but this formalism is too advanced for students at the secondary school level. The presentation is therefore often limited to some key concepts and phenomena, which often include the duality descriptions of light and associated experiments. Krijtenburg-Lewerissa, Pol, Brinkman, and van Joolingen (2017) remarked that the conceptual approaches to learning quantum physics developed in recent years have made it possible to introduce these topics also in the secondary school level in several countries.

Quantum physics, and especially its philosophical implications, does not traditionally have an extensive place in school physics internationally. Comparing a handful of upper secondary physics curricula in Western countries, Henriksen et al. (2014) noted that the wave-particle duality of light is a very common topic, along with the photoelectric effect. Phenomena such as entangled photons, or explicit mention of interpretations of quantum physics or philosophical implications, are rare. In France, quantum physics was reintroduced in the curriculum for upper secondary school physics in 2012 after 20 years of absence, including among other topics the photoelectric effect, lasers and wave-particle duality (Lautesse et al., 2015). This new curriculum in France is of special interest because instead of presenting the wave-particle duality according to the Copenhagen school, the curriculum points out a different innovative position where a photon is described as a pure quantum object that should be compared with neither a particle nor a wave. This position is in accordance with Mario Bunge's quanton concept mentioned above. However, in French textbooks the term quanton is rarely used.

In Norway, where the present study was carried out, the upper secondary physics curriculum emphasises a qualitative understanding of central concepts and philosophical aspects of physics. The curriculum (NDET, 2006) states that students in the highest-level physics course in year 13 should be able to:

- give an account of Einstein's explanation of photoelectric effect, and give a qualitative account of how results from experiments with photoelectric effect, Compton scattering and the wave nature of particles represent a break with classical physics

- give an account of Heisenberg's uncertainty relations, describe the phenomenon "entangled photons" and give an account of their cognitive (epistemological) consequences ${ }^{1}$

Consequently, in this field of physics the curriculum in Norway does not require that students perform many calculations, but rather that they give qualitative descriptions and are able to discuss philosophical and epistemological aspects of physics including aspects of "the nature of science" (NOS).

NOS refers to the epistemology of science, science as a way of knowing, or the values and

\footnotetext{
${ }^{1}$ We believe that «epistemological consequences» is a better translation of the Norwegian terms than «cognitive consequences», which is the phrase used in the Ministry's official English translation of the curriculum.
} 
beliefs inherent in the development of scientific knowledge. NOS is that element of the science curriculum in which students learn how science functions, how scientific knowledge is generated and tested, and how scientists do their work (McComas, Almazroa, \& Clough, 1998). A widely recognized interpretation of the nature of science includes that scientific knowledge is tentative, empirical, theoryladen, partly the product of human inference, imagination, and creativity, and socially and culturally embedded (Lederman, Abd-El-Khalick, Bell, \& Schwartz, 2002).

Internationally, there have been many suggestions during the last decades that science education should give students a better understanding of the nature of science. For example The Next Generation Science Standards in the USA state:

Epistemic knowledge is knowledge of the constructs and values that are intrinsic to science. Students need to understand what is meant, for example, by an observation, a hypothesis, an inference, a model, a theory, or a claim and be able to distinguish among them (NGSS, 2013).

This is in line with McComas et al. (1998), who stated that NOS must be a clear learning goal given prominence in the classroom along with science content, and that negotiating sophisticated epistemological understandings can be handled by students on upper secondary level. Also Abd-ElKhalick (2012), when discussing the question of benchmarking understanding of NOS, emphasised that learners in secondary school would be expected to discuss NOS aspects with reasonable sophistication. The Norwegian curriculum is in line with these internationally recognized ideas, since it also states that students should be able to (NDET, 2006):

- elaborate on and discuss how various theories of physics can exist side by side, even though they are contradictory

- give an example of a scientific conflict that has been resolved and how, and an example of a scientific conflict that remains unresolved and why.

An important aspect of the nature of physics is an understanding of the scientific enterprise as producing models of our physical reality. Gilbert (2004) pointed to the importance of students' understanding of the nature and significance of models within the development of science. He wrote that "models are essential to the production, dissemination, and acceptance of scientific knowledge" and that they "function as a bridge between scientific theory and the world-as-experienced ('reality')" (Gilbert, 2004, p. 116). Treating the wave-particle duality of light provides an opportunity to discuss epistemological aspects of physics. This includes for instance the role of models, the role of controversy in the development of science and the philosophical underpinnings of scientific models. Cheng and Lin (2015) wrote that many students have a naïve-realist view of scientific concepts, and do not distinguish between target events in the real world and entities created for the purpose of model-building. Furthermore they accentuated that secondary students have a better understanding of the process of modelling than of the nature and purpose of models. They suggested as implications for teaching "...that the ontological distinction and sophisticated relationships between models and the target events should be taught and emphasized explicitly in the curriculum, when students learn modelling and understanding of scientific models" (Cheng \& Lin, 2015, p. 2470).

The Norwegian curriculum, with its emphasis on philosophical aspects, provides opportunities as 
well as challenges for an innovative teaching of modern physics_(Henriksen et al., 2014). These curriculum goals are the starting point for the development of the online learning resources we describe later in this article.

\subsection{Previous research on teaching and learning about quantum physics and the} nature of light

Research on students' understanding and learning in quantum physics is relatively scarce compared to other fields of physics. The studies undertaken have mostly focused on common difficulties for undergraduate students at the university level (Krijtenburg-Lewerissa et al., 2017). Very few studies address how pre-university students recognize aspects of ontology and epistemology in the context of quantum physics, and here we present research on undergraduate as well as upper secondary physics students.

Karakostas and Hadzidaki (2005) pointed to students' cognitive structure as characterized by a rather classical perception of quantum physics. For example, students transfer macroscopic attributes to sub-microscopic objects and transfer a deterministic mode of classical reasoning into the quantum physics context. To avoid this confusion, Hadzidaki (2008) argued that students need to explicitly be made aware of how quantum physics breaks with the principles of classical physics. Greca and Freire (2003) tried to identify some key ideas that students use to understand concepts related to the microscopic world. Among engineering students they found that most of them had varieties of classical ways of visualization, and to most of them the quantum concepts were fragmentary or mere mathematical expressions. They wrote:

These results indicate that traditional approaches in introductory QM courses present scarce success: none of the concepts usually considered essential to describe quantum world seem to have been adequately understood by most of students (Greca \& Freire, 2003, p. 546).

Furthermore they suggested a teaching/learning strategy applying a phenomenological-conceptual approach and avoiding classical analogies, and they reported favourable outcomes in terms of learning and motivation when using the strategy with three groups of engineering students.

Baily and Finkelstein (2010b) reported differences in how quantum physics instructors in undergraduate physics courses differed in their treatment of interpretations of quantum physics. The instructors' interpretations have impact on student thinking, and Baily and Finkelstein (2010b, p. 9) concluded:

In the end, we argue that modern physics instructors should concern themselves with matters of interpretations, if only because their students concern themselves with these matters, and as educators we should be concerned with what our students believe about physics and the nature of practicing physics.

A survey by Olsen (2002) reported on challenges in Norwegian upper secondary physics students' understanding of concepts such as wave-particle duality of light. He found that the waveparticle duality was poorly understood, and some students clearly demonstrated misconceptions rooted in a classical physics worldview. Renstrøm (2011), after a study of how the historical development of 
quantum physics was presented in Norwegian textbooks and how it was interpreted by students, recommended teaching explicitly how quantum physics breaks with classical physics and using the historical development to support students' conceptual development.

Also Ireson (2000) showed that many upper secondary school students mixed the wave and particle descriptions of light. He argued that concepts such as wave-particle duality and matter waves should be avoided, and that photons and electrons should be described as quantum objects rather than as particles or waves, in line with the conception of quantons suggested by Bunge (2003). In contrast, Cheong and Song (2014) recommended using duality in quantum physics teaching, in particular as a starting point for discussing interpretations of quantum theory. They described three levels of meaning of duality. On the first level, duality is not related to other core concepts of quantum theory and is not related to the formalism in quantum physics, but this level includes that light has both a particle property and a wave property, relating this to experimental results. The second level includes "core ideas of quantum theory such as the wave function, Born's probabilistic interpretation, Schrödinger's equation, superposition principle, and the uncertainty principle" (p. 1016). The core ideas are considered theoretical tools and "concepts are merely used to predict phenomena and any claim of reality is not contained in the meaning of this level" (p. 2017). In the third level of the meaning of duality, Cheong and Song included conflicting interpretations about the status and role of quantum theory, and they stated that "in accordance with one's philosophical position, the ultimate purpose of science could be merely predicting (or explaining) phenomena or making a reality claim on the hidden structure or process of nature. Thus, both purposes must be distinguished in any discussion on the nature of scientific knowledge" (p.2017). Cheong and Song (2014) emphasized that different levels of meaning of duality should be taught on appropriate stages in physics education. For example, introductory courses should focus on light as having both a particle property and a wave property, without relating duality to core formal concepts such as wave function or superposition.

Gjerland (2015) analysed results from upper secondary classroom trials of an earlier version of the teaching resources described in the next section. He found that about half of the upper secondary students in the sample had a reasonable understanding of duality corresponding to the first level of duality as described by Cheong and Song (2014), but also that some students displayed an uncritical duality notion. This means that they combined wave and particle properties, for example by describing light as "particles with wavelength", without reflecting on the contradictions between these properties in a classical sense. He also pointed out that while many students were able to describe light as displaying both wave and particle properties, some associated the wave description solely with classical physics and failed to acknowledge that a wave description is also inherent in the quantum description of light as photons. Along similar lines, Cheong and Song (2014, p. 1027) remarked that the photon model "hides the wave-like nature of the photon by merely focusing on its particle-like nature" and recommended that care should be taken with using appropriate language when treating this topic in instruction.

Krijtenburg-Lewerissa et al. (2017) presented a review of the current state of research on teaching quantum mechanics in secondary and lower undergraduate education. They concluded among other things that many challenges that students experience are related to the inability to connect quantum behaviour to the physical reality as they see it, which results in a mix-up of classical and quantum concepts. Referring to Ireson (1999; 2000) and Ayene, Kriek, and Damtie (2011), they 
presented three clusters of students' understanding of wave-particle duality: “(1) Classical description, in which students describe quantum objects exclusively as particles or waves; (2) mixed description, in which students see that wave and particle behaviour coexist, but still describe single quantum objects in classical terms; and (3) quasiquantum description, in which students understand that quantum objects can behave as both particles and waves, but still have difficulty describing events in a nondeterministic way" (Krijtenburg-Lewerissa et al., 2017, pp. 2-3).

Mannila, Koponen, and Niskanen (2001) studied undergraduate students' conceptions of waveand particle-like properties of quantum entities based on double-slit interference. They found that classical ontology and trajectory conceptions were dominant in many responses, and claimed that the main difficulty in students' conceptions of wave-particle duality is related to a need for a new ontology.

Baily and Finkelstein have discussed undergraduate students' perspectives on quantum physics in several papers (Baily \& Finkelstein, 2009, 2010a, 2010b) and have particularly addressed issues of interpretation. For example, they found (Baily \& Finkelstein, 2010a) that some students differentiated between what made intuitive sense to them personally (often a realist perspective) - and what they believed an expert physicist would say (a probabilistic and non-local description, often a Copenhagen interpretation). The same authors observed that students were more likely to prefer a realist interpretation of quantum physics when the teachers were less explicit in addressing student ontologies, and they suggested that teachers should address questions of ontology in quantum physics explicitly across topics (Baily \& Finkelstein, 2010b). In discussing whether students hold their own ontologies (defined as "mental categories of concepts, substances and processes") in quantum mechanics, Baily and Finkelstein (2014) claimed that students may flexibly switch between ontological categorisations of quantum objects and create blended ontological categories such as that of wave and particle. Moreover, they found that students' conceptions of quantum objects were modified across situations, often in a non-consistent manner, and they recommended that instructors explicitly help students to consciously separate classical and quantum ontologies.

To sum up: Students tend to mix classical with quantum physics notions when trying to conceptualise light and other quantum phenomena. This is probably related to an insufficient understanding of how quantum physics breaks with some central assumptions in classical physics and to teaching not addressing issues of interpretation.

\subsection{The ReleQuant learning resources for upper secondary physics}

Within the project ReleQuant we have developed online research-based learning resources in quantum physics and general relativity for upper secondary school physics (Bungum, Henriksen, Angell, Tellefsen, \& Bøe, 2015; Bøe, Henriksen, \& Angell, in press; Henriksen et al., 2014). Resources have been designed through several cycles of development, school testing and modifications, as prescribed by a DesignBased Research methodology (Anderson \& Shattuck, 2012). The project has involved the collaboration between physicists, physics education researchers, physics teacher students and practicing teachers. Qualitative understanding and historical-philosophical aspects of modern physics are emphasized in the resources, in line with the Norwegian curriculum for upper secondary physics. The research objectives of ReleQuant are to study how the learning resources function in classroom settings and to get insight into 
students' motivation, conceptual understanding and learning processes in modern physics. ${ }_{-}^{2}$ The recourses are available through viten.no, a platform run by the Norwegian Centre for Science Education.

ReleQuant is based on a sociocultural view of learning, where conceptual understanding is seen as a result of social interaction and the use of language (Vygotsky, 1978). Thus, the resources let students explore physics concepts through writing tasks and "talking physics" (Henriksen \& Angell, 2010) and discussing in peer groups (Bungum, Bøe, \& Henriksen, 2018). Through the digital platform, students give written responses to questions in the learning platform where the teacher can view and comment on each student's response, and the students are challenged to discuss in pairs and to participate in whole-class discussions.

In the ReleQuant material on quantum physics, the first section is called Need for a new physics. It uses the nature of light as a starting point for working with the historical development of quantum physics and how it constitutes a break with classical physics. The students start with answering the question "What do you think light is?" in writing, based on their previous knowledge. After this follows a short animation video about the development of quantum physics, focusing especially on the nature of light, Einstein's work on the photoelectric effect, his photon hypothesis and Niels Bohr's initial opposition. The video ends up stating that we still have two models for light, the particle model and the wave model, and that physics in general is still an ongoing endeavour. Another video follows, where two research physicists (one of them among the authors of this article) answer the question "What is light?" in quite different ways. The theoretical physicist explains it from a typical wave-particle duality perspective, primarily advocating the particle model. He does, however, also state that light is neither traditional waves nor particles, but a quantum object of its own. The other physicist sticks to the wave model, and explains how he is critical of the wave-particle duality in quantum physics, and points, for example, to the paradox in that a point particle can be assigned a wavelength.

The students are then asked to discuss in pairs the question "Is it possible to think of light as being both wave and particle?". This discussion task was introduced in the learning resource based on earlier results from the project (Gjerland, 2015) showing that students may hold an uncritical duality view, describing light as waves and particles without reflecting on the contradiction this represents on an ontological level. After this discussion task, a short video follows where the same two physicists talk briefly about how their differences of opinion boil down to interpretations of quantum physics and philosophical points of view, focusing on the Copenhagen interpretation versus a realist philosophical stance.

In an introduction to the ReleQuant material, students are told that they will work with physics in ways that may differ from their usual physics lessons (Bøe et al., in press). They will use written and oral language more than calculations; they will work with questions without clear and unambiguous answers; they will practice thinking and making arguments like physicists, and they will see historical as well as contemporary examples of how physics progresses through discussion and controversy. In this way, students are made aware that they are expected to learn something about the nature of science as well as acquiring a conceptual understanding of light and quantum physics.

${ }^{2}$ http://www.mn.uio.no/fysikk/english/research/projects/relequant/index.html. The ReleQuant learning resources are available in English https://www.viten.no/eng/ (a later version than the one studied in this paper). 


\subsection{Aim and research questions}

The empirical study reported in this article investigates how upper secondary physics students conceptualise the nature of light. We use this to explore to what extent and in what ways it is feasible to introduce ontological and epistemological perspectives on the scientific description of light in upper secondary physics. The empirical study is guided by the following research questions:

1. Which conceptions about the nature of light do upper secondary physics students express through an individual writing task at the beginning of a teaching sequence about quantum physics?

2. How do upper secondary physics students conceptualise the wave and particle nature of light through small-group discussions after having worked with a short teaching sequence addressing problematic aspects of the wave-particle duality of light?

3. What epistemological reflections and conceptions about the nature of science (NOS) do student responses reveal?

\section{Method}

\subsection{Sample and data collection}

In Norway students can take an optional physics subject in the two final years (year 12 and 13) of upper secondary school. About 13-14 \% of the total age cohort choose physics in year 12 and 6-7 \% in year 13 . This article builds on data collected in March - May 2016 from 188 final-year students (18-19 years old) in 9 physics classes in 7 upper secondary schools in southern Norway, most of them in the region around the capital, Oslo. These schools are regarded as having middle to high-achieving students in national comparison. Students were taught quantum physics by their regular teacher, using the ReleQuant online resources described above. The teachers of all these students had attended at least a two-day seminar introducing the ReleQuant material and including lectures on quantum physics topics. The sequence about light appears at the very beginning of the quantum physics unit.

In this article, we analyse two sets of student data:

1. Individual, written responses to a question posed at the very beginning of the quantum physics material (Fig.1): "What do you think light is? Describe it as well as you can. Use three to four sentences."

2. Transcripts of recordings of small-group discussions of the following problem (Fig. 2): "Discuss with a fellow student: Is it possible to think of light as being both wave and particle?" 


\section{Start-up writing task}

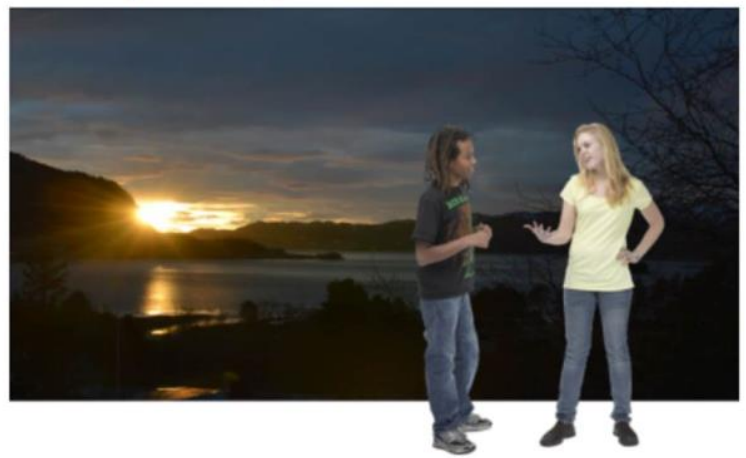

Both in classical physics and in quantum physics we find descriptions of light, and the question of what light is, was important for the development of quantum physics.

What do you think light is? Describe it as well as you can. Use three to four sentences.

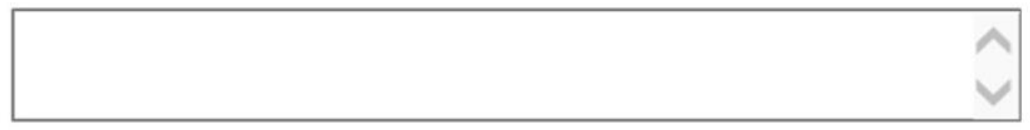

Fig. 1 Screenshot from the $\mathbf{2 0 1 6}$ version of the ReleQuant learning resources: Individual writing task at the start of the quantum physics sequence

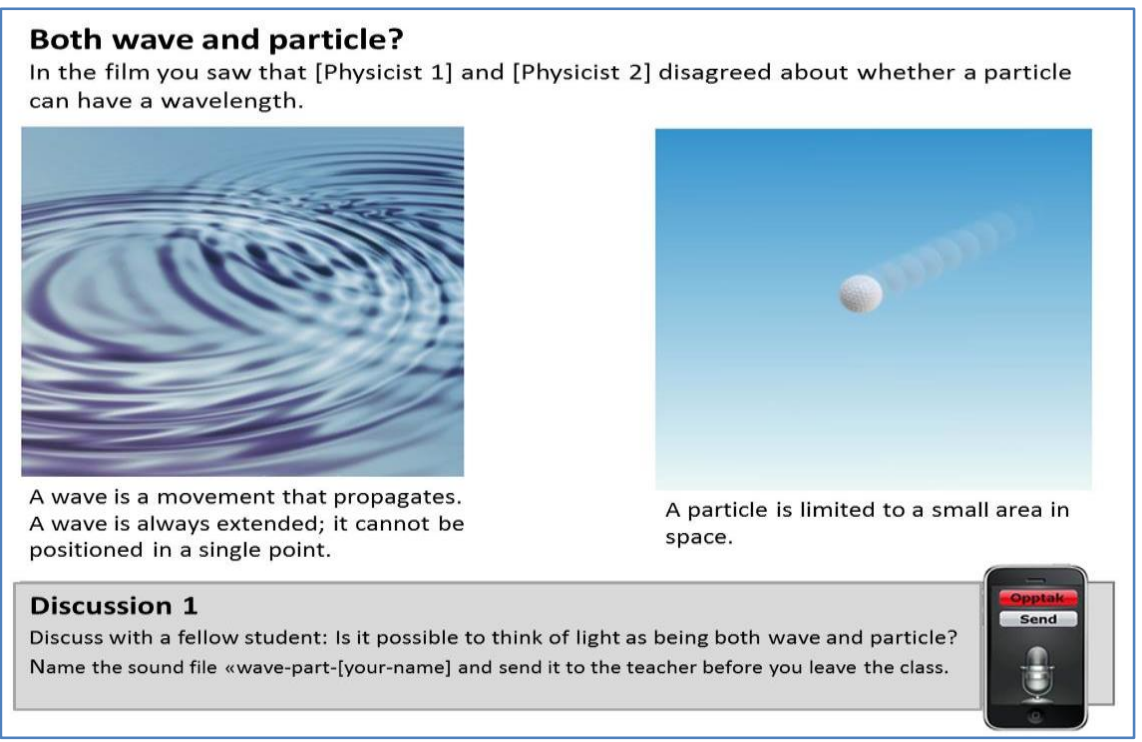

Fig. 2 Screenshot from the 2016 version of the ReleQuant learning resources: Smallgroup discussion task about the wave and particle nature of light, given after students have watched a video addressing problematic aspects of the wave-particle duality

The individual, written responses (1) were collected through the online learning platform where the ReleQuant material is presented. A total of 133 written responses to the question were received.

Students recorded small-group discussions (2) on their own smartphones and sent them to their teacher, who forwarded them to the research team. Research assistants then transcribed the discussions. The analysed material consisted of a total of 55 discussions involving 2-3 students each; thus, a total of at 
least 120 students contributed to the analysed recordings. Each recorded discussion lasted from a few seconds up to around five minutes. Both the students and the teachers had given their consent to take part in the research project.

\subsection{Analysis}

Students' responses (written and oral) were analysed qualitatively using a thematic approach (Braun \& Clarke, 2006) to identify patterns and themes within student responses. The software ATLAS.ti was used to code, sort and retrieve quotations.

For analysis of the written responses (Fig. 1), two of the authors first coded the entire material through a mainly inductive process, but keeping in mind the research questions as well as the challenges presented in the Introduction to this paper such as a wave versus particle descriptions of light; acknowledgement of the ontological paradox with the two models; philosophical perspectives, and understanding of the nature of science. This coding was conducted mostly independently, but with some discussion along the way. After this preliminary coding, authors went back to the published literature, discussed preliminary codes and tentative findings in light of this, then discussed and agreed on a common set of codes. The entire material was then coded again by the same two researchers through a collaborative process where interpretations of the material were discussed and agreed on (see Table 1 in the next section). Several codes were assigned to the same sequences where appropriate.

Analysis of transcripts of small-group discussions (Fig. 2) was done in the same manner (see Table 2 in the next section). Some codes were common to both analyses, whereas others were unique to each data set.

All excerpts and quotes presented in the Results sections have been translated to English by the authors. Some of the oral quotes have been lightly edited to aid readability; usually by omitting expressions like "..er..." in cases where they do not seem important to conserve meaning.

\section{Results}

\subsection{Student conceptions about light, expressed through an initial, individual writing task}

The 133 written responses to the question "What do you think light is?" were numbered from 1-133, and student quotes are presented with numbers (e.g. S 4, S 89, etc). The codes assigned to the responses are presented in Table 1. 
Table 1 An overview of the codes used in analysing students' written responses $(N=133)$ to the question "What do you think light is?". The number of responses to which each code was assigned is also indicated.

\begin{tabular}{|l|l|l|}
\hline Code & Characteristics & Number \\
\hline Wave and/or particle & Dual nature of light & 61 \\
\hline Photons/energy packages/quanta & Emphasising the particle nature of light & 51 \\
\hline Energy & Light as being, containing or carrying energy & 32 \\
\hline Electromagnetic radiation/waves & Light described as electromagnetic radiation/waves & 28 \\
\hline Speed of light & Properties of light such as its speed & 25 \\
\hline Visible wavelengths & Wavelengths visible to the human eye & 25 \\
\hline Excitation & Light emitted from atoms - the Bohr model & 20 \\
\hline Light is waves & Emphasising the wave nature of light & 16 \\
\hline Photon wave properties & Photons/particles described with wave properties & 16 \\
\hline Gravitational bending & Light can be bent in gravitational fields & 10 \\
\hline Something else & The true nature of light may still not be known & 6 \\
\hline Depending on experiment & $\begin{array}{l}\text { Light as displaying wave or particle nature depending } \\
\text { on the situation or experiment. }\end{array}$ & 5 \\
\hline Particles in wave-shaped pattern & $\begin{array}{l}\text { Photons are moving like waves or in a wave-shaped } \\
\text { pattern }\end{array}$ & 3 \\
\hline Other & Other codes used, but few responses for each code & 8 \\
\hline
\end{tabular}

As Table 1 shows, light as wave and/or particle was the most frequent description (found in almost half of the responses). Various formulations were used to express this dual nature of light: Light is or can be both waves and particles; light can behave as both waves and particles; light can be seen or be described as, or consists of waves and particles:

- I think that light is waves and particles (S 20)

- Light behaves as both particles, rays and waves (S 90)

- Light is, usually, described either as a wave or as small packets of energy, photons (S 59)

These formulations clearly reflect what students have been taught in previous physics teaching. Even if the responses are short, a realist view can be detected in how some students expressed their knowledge as what light is, as is the case with the first response quoted above. Many students also expressed an understanding of that scientific models are human descriptions of reality, for example by wording like "behaves as" and "described as" in quotations above.

Many students started out with a clear wave or particle statement, but soon proceeded to a duality perspective:

- $\quad$ Light is a stream of photons. It has both wave properties and particle properties (S 41)

- $\quad$ Light is electromagnetic radiation which can behave both as waves and as particles. (S 28)

A handful mentioned light as displaying wave or particle nature depending on the situation or experiment. 
- Depending on which tests we perform, light can come in the form of either particles or waves. (S 23) Very few responses questioned or commented on how these two conceptually different models could describe the same phenomenon, but a handful emphasized that the true nature of light may still not be known to science.

- [Light] has both particle properties and is electromagnetic waves in some magical way. (S 30)

- I don't think we really know what light is, but we use what we know to define something we cannot really explain. Therefore we say that light has both the properties of particles and waves. Something is lacking, anyhow, even if some of what we use to explain it, is correct. (S 37)

Again, a realist perspective seems to be inherent in this expression.

A large group of respondents (more than 1/3) emphasised the particle nature of light, although some of them also mentioned waves later on in their response. Responses coded as emphasising the particle nature of light contained terms such as particles, photons, energy packages/portions, and quanta.

- $\quad$ Light is small particles/photons that are emitted by atoms. (S 6)

- Light is photons. Photons have both the properties of wave radiation and particle radiation. (S 36) A somewhat smaller group of respondents emphasised the wave nature of light or described it as electromagnetic radiation.

- Light is waves moving through space at the speed of light. (S 43)

- Light is electromagnetic radiation. It moves in waves. (S 39)

A common formulation was also light as being, containing or carrying energy.

- Light is a form of energy transport (S 10)

- The waves consist of energy... (S 34)

- $\quad$ Light contains energy. (S 65)

Many responses mentioned properties of light such as its speed, which wavelengths are visible to the human eye, how light is emitted from atoms (typically referring to the Bohr atomic model), or that it can be bent in gravitational fields.

- It has a speed of $300000000 \mathrm{~m} / \mathrm{s}$, also known as c (S 12)

- $\quad$ Light is photons, "energy packets", that are sent out from atoms when electrons jump into shells closer to the atomic nucleus. (S 47)

- Light cannot have mass but is nevertheless deflected by massive objects. (S 42)

These aspects were probably mentioned due to what students had been exposed to earlier through the general science and physics curriculum. Most students had recently been through the topics of special and general relativity; hence the focus on the speed of light and on how light is bent around massive objects. The general science curriculum in year 11 , as well as the physics curriculum in year 12 , treats the Bohr model of the atom and how photons of certain wavelengths are associated with transitions between energy levels. 
A few responses exposed the misconception that photons are moving like waves or in a waveshaped pattern.

- $\quad$ Light is a kind of moving particles where photons are sent out in rays/waves ...(S 82)

Many of the responses were characterised by an imprecise use of terminology, using terms relevant to a description of light, but in a way that is not meaningful in a strict physics context. Several of these responses include statements indicating that photons or particles have wave properties.

- $\quad$...light moves as particles with waves... (S 29)

- Photon particles that function as wave particles (S 129)

This faltering use of terminology may have many reasons, ranging from Norwegian not being the student's first language or a misunderstanding of specific terms, to a deeper conceptual confusion.

Finally, it should be mentioned that several students gave quite comprehensive answers, for example:

- Light can be described both as waves and as particles (photons). If viewed as waves, we call visible light electromagnetic radiation with wavelengths between $380 \mathrm{~nm}$ and $750 \mathrm{~nm}$. When we talk about the light we get due to exciting electrons in atoms, we use the particle model. The atom then releases a photon with a certain amount of energy when it goes down to a lower energy level again. (S 80)

This quote illustrates that at least some of the students were able to express important aspects of what light is even before they started the work with the ReleQuant recourses.

\subsection{Students' conceptualisation of the wave and particle nature of light through small-group discussions after a teaching sequence}

As described above, the discussion task about the wave and particle nature of light was given to students after they had watched a 2-minute animated video summarizing the history of quantum physics and a 5-minute video where two physicists discussed interpretations of the wave and particle nature of light. The two physicists displayed different viewpoints on the nature of light, and results show that many of the small-group discussions appeared to be influenced by these presentations.

Table 2 gives an overview of the codes used in the analysis of the 55 group discussions and a brief characterisation of each code. The number of conversations to which each code was assigned is also given. 
Table 2: An overview of the codes used in analysing the $\mathbf{5 5}$ student small-group discussions of the question "Is it possible to think of light as being both wave and particle?". The number of groups to which each code was assigned is also indicated.

\begin{tabular}{|l|l|l|}
\hline Code & Characteristics & Number \\
\hline Depending on experiment & $\begin{array}{l}\text { Light as displaying wave or particle nature depending on } \\
\text { the situation or experiment. }\end{array}$ & 28 \\
\hline NOS & $\begin{array}{l}\text { Aspects of the nature of science and epistemological } \\
\text { reflections }\end{array}$ & 17 \\
\hline Recognition of the paradox & $\begin{array}{l}\text { Reflection on the paradox that light is described as both } \\
\text { localised (as particle) and extended (as wave) }\end{array}$ & 15 \\
\hline Wave AND particle & Expressing that light is both wave and particle & 14 \\
\hline Reference to videos & Explicit references to the physicists in the videos & 13 \\
\hline Something else & $\begin{array}{l}\text { The true nature of light may still not be known and a } \\
\text { new theory for light is needed }\end{array}$ & 12 \\
\hline Particles in wave-shaped pattern & $\begin{array}{l}\text { Photons are moving like waves or in a wave-shaped } \\
\text { pattern }\end{array}$ & 11 \\
\hline Other & Other codes used, but few responses for each code & 18 \\
\hline
\end{tabular}

In the excerpts from discussions presented in this chapter, groups are labelled Group 1 - Group 55, and students within each group are labelled G1-S1; G1-S2, and so on. In the discussions about whether it is possible to think of light as being both wave and particle, the most commonly occurring theme was light displaying either particle or wave properties depending on the situation or experiment (Table 2). For example:

\section{Group 40:}

G40-S1 - Is it possible to think light as being both wave and particle?

G40-S2 - Yes, I would say so. To explain two, or more, different phenomena related to light, one has to use several models. For instance, one can support the particle model with photoelectric effect and the wave model with interference patterns. Since we haven't come up with any other explanation, we just have to accept that light behaves as both particles in some cases and as waves in others. So yes, it is possible and necessary.

\section{Group 6:}

G6-S1 - So from what we have seen here, then, the two are sort of opposing, then; a wave cannot be in a point, a particle is in a point.

G6-S2 - Yes

G6-S1 - So in that way it seems really strange to think, but then we have seen in experiments that it can have both wave properties in that it for instance ... interference, and it has particle properties as in photoelectric effect.

The responses resemble formulations that may be found in textbooks and what students saw in the video in the teaching material. Students were able to refer to waves and particles as models for light, and to specific experimental evidence for each model. In the first group referred above, student S2 
concluded that it is necessary to have two models for lack of a better description, while in the latter group student S1 considers also the underlying reality in expressing that it is "really strange" that light can have opposing properties.

To help students move beyond an uncritical duality description of light, an explicit aim in developing the ReleQuant resources was to have students reflect on the inherent paradox in light being described as both localised (as particle) and extended (as wave), and about the meaning of a particle (the photon) being assigned a wavelength. This was explicitly discussed in the video. Compared with the written answers to the start-up task, a much greater proportion of responses now contained explicit reflections on this paradox.

\section{Group 2:}

G2-S1 - But it's also like, like when he talks about particles that have wavelength, its completely incomprehensible ...

G2-S2 - Yes

G2-S3 - It becomes at least very abstract

G2-S1 - yes, it's only one point, so how can it have a wavelength?

\section{Group 20:}

G20-S1 - yes, but light ... if it is both a particle and wave we can see that particles and waves are defined in different ways where particles are points and waves are extended. So how can something be both a point and something extended?

Inspired by the material presented in the videos, these students were able to express interpretational challenges in the wave-particle duality of light beyond an uncritical duality view, perhaps also touching on ontological questions concerning the wave and particle description of light.

Some of the groups tried to conceptually reconcile the wave and particle descriptions, in certain cases resulting in the misunderstanding that photons move in a wave-shaped pattern or trajectory. This is illustrated in the following example:

\section{Group 8:}

G8-S1 - I think it is strange what he said about particles having wavelength, how can a particle have wavelength?

G8-S2 - No, l, find that weird, too (...) because we have learned that light is particles, or wavelength.

G8-S1 - M-hm

G8-S2 - But that becomes, sort of, a particle with wavelength (...) that is weird

G8-S1 - Like the question says, then - both wave and particle, is that possible?

G8-S2 - Yes, ehhm...

G8-S1 - Maye it can make itself into both wave and particle, then (giggles)

G8-S2 - Or maybe it is just particles that come in a wave shape

G8-S1 - Yes, yes, maybe, they come sort of in a long row

G8-S2 - They are rows that have waves 
G8-S1 - But what I really think, then, is that there are just certain things that are impossible to explain, and that, that is such a thing that just doesn't fit into any of those explanations, so there must be an entirely new....

G8-S2 - That may be...

G8-S1 - ...theory, and we cannot explain it with words or pictures or anything because that is just how it is, like

G8-S2- True, it might be that none of those are quite in accord with those theories

G8-S1 - Yes, and what I think, then (...) there is a long, long row of particles, so they become wavy.

G8-S2 - Yes, we can have that as our answer, but then again, it is hard to be certain.

Although these two students ended up concluding with a misunderstanding about a row of particles in a wavy pattern, they had a noteworthy discussion where they express their dissatisfaction with the two models and are looking for something entirely new.

Several of the groups attempted this kind of conceptual reconciliation between a wave and a particle model:

\section{Group 18:}

G18-S1-Yes, I don't know, but I thought that for me it is hard to envision that something can be in waves without being sort of a physical thing. So it is easier to see a particle or a point that moves in a wave pattern.

\section{Group 33:}

G33-S1 - Can something be two things at a time really? Two so different things. If it isn't a wave of particles. Because in that case it can be both things, since then it can look like a wave if we look at it from a distance

In the latter utterance, the student targeted the issue of simultaneousness. Does the duality mean that a photon is wave and particle at the same time, or that it changes properties according to the context? This is touched upon also in a few other student groups, for example:

\section{Group 32:}

G32-S1 - We should use the explanation that fits best every situation.

G32-S2 - Yes

G32-S1 - But the question is really if it can be two things simultaneously. Or if it really is two different things.

Here it is worth noting how the student's formulation "if it really is two different things" clearly reveals a realist view where a reality exists independently of our models and measurement, even if the same student also shows a more instrumental approach in the beginning of the quoted sequence, in asserting that we should use the explanation that fits best every situation.

Other challenges students encounter are related to how we can understand light as a wave. In the following dialogue, this leads to quite extensive speculations:

\section{Group 21:}

G21-S1 - The question is: When you have the waves, what is at the end of the wave, what is at the front of the wave, sort of? Is it a particle or is it just nothing? Is it the same in the entire... 
G21-S2 -Maybe a wave is just a really long particle. A snake particle.

G21-S1 - That is this string theory thing, you know

G21-S2 - Yes

G21-S1 - It is both a wave and particle thing in a... string

G21-S3 - Yes

The students' starting point in the above dialogue was how to envision a wave. What is at the end and at the front of a wave? This problem may be related to students' experiences of waves in everyday life, where waves on water may be produced by a boat or a stone, which are then in the "end" of the wave, or waves on a string or a rope, where the end points must be attached to something physical that makes the wave motion possible. Some students expressed that sound waves are easier to comprehend, since the material substance that vibrates is clear to them, for example:

\section{Group 1:}

G1-S1 Because, kind of, photons are small packets of energy in a way, but waves ... that ... what is that, sort of, what kind of wave is it, is it just like propagation in, like, the particles in the room, kind of?

(...)

G1-S2 Sound waves make sense, but light waves don't quite make sense.

G1-S1 Yes, because sound waves they vibrate and stuff, and the eardrum vibrates and ... all that there.

It seems from these results that a main challenge students had in comprehending light as waves is that they lacked a conception of what is "waving". One student in the sample got around this challenge by describing the wave as a probability distribution:

\section{Group 22:}

G22-S1: The way I see it's like, the wave is just a representation of where it is most likely that the particle is, and that, there is a, the higher the wave crests and the lower the frequency, it means that there is more energy, which means there's a better chance of a photon coming ... being created.

Some discussions, clearly inspired by the teaching sequence, expressed the view that the true nature of light has still to be described by science and that a new theory for light was needed in order to resolve the conceptual paradox:

\section{Group 14:}

G14-S1 - Yes, I think there must be some other way of explaining it, that lets us have... that explains both the waves, both as a particle and as a wave, then. Yes, some other common way to explain it, that we haven't understood.

\section{Group 3:}

G3-S1 - Oh, so it could be this, that in the future one will find out that it is really the same.

G3-S2 - M-hmm

G3-S1 - But one can say it in different ways

G3-S3 - Yes, anyway something is missing in the models that can make us understand it

This last student expressed a wish for some kind of conceptual model or visualisation - not primarily a better scientific model, but something that can make the scientific description intelligible for him personally. 


\subsection{Epistemological reflections and conceptions about the nature of science expressed through student discussions}

Aspects of the nature of science (NOS) and epistemological reflections were present in around $1 / 3$ of the students' discussions (Table 2). Some of the discussions referred to expectations for science to prove that one or the other model of light was correct, as shown in these examples:

\section{Group 5:}

G5-S1 -Yes, because he (the physicist in the video) who was in favour of waves, tried to prove that those experiments (...) that worked if light was photons, that those also worked for waves.

(...)

G5-S2 - You could say that light is both particles and waves, but it is hard to prove for both

\section{Group 27:}

G27-S1 - Isn't it so that quantum physics hasn't been proven?

G27-S2 - That it is just a theory?

G27-S1 - That it is just a theory, yes.

G27-S2 - A lot of things are just theory, so that makes sense

These students expressed a somewhat naïve view of the scientific process, expecting science to provide definite proof of claims about some underlying reality. Their use of the phrase "just a theory" perhaps reflects everyday uses of the phrase "theory" as an (educated) guess, in contrast to the scientific sense of "theory" as a comprehensive framework of empirically and rationally supported laws, models or claims about the natural world.

Other groups expressed a notion of physics knowledge as models making it easy to explain or predict phenomena, in some cases expressing an instrumentalist approach:

\section{Group 23:}

G23-S1 - It doesn't say in the explanations that we just read that light was a particle, that it behaved like a particle, it was just easier to explain a phenomenon, so we don't have any concrete evidence for it, as far as I know

\section{Group 28:}

G28-S1 - Yes, it is a very interesting discussion. It is a bit contradictory that we have two so different models to explain the phenomenon of light. But yes, I don't know...

G28-S2 - I assume that it is OK as long as you get a better description of it, given that you don't have one, that is, a description that you are more certain that you cannot prove totally, then I assume it is OK with both models for the time being

\section{Group 48:}

G48-S1 - so I think the photon explanation is just a simplification to make it easier to imagine what it is, and to, yes, give a picture that is not necessarily correct, but only a way to explain 
This student appeared to view the photon model most of all as a pedagogical tool devised to make the physical theory intelligible, a view also expressed in the following exchange:

\section{Group 22:}

G22-S2 - If light doesn't have mass (...) Why is it always illustrated as an object (...)?

G22-S1 - It is only so that people can understand, it is only to make them understand it ...

However, the knowledge produced in physics could also be seen as an insufficient representation of reality:

\section{Group 34:}

G34-S1 - So none are perfect understandings then

G34-S2 - That is a good way of saying it

(...)

G34-S1 - So we do not really know the truth, we only know an approximation to it in a way G34-S2 - Yes.

These students, although they use the word "truth", appear to have a view of science that goes beyond the "naïve-realist epistemology in which models are perceived as physical copies of target phenomena" (see Cheng \& Lin, 2015).

Others acknowledged how quantum physics can deliver mathematically based predictions, but expressed disillusionment concerning the ability of science to come up with models or descriptions that are possible to imagine conceptually:

\section{Group 31:}

G31-S1 - ...but the question is; is it possible to imagine that light is both wave and particle. And that's possible, that's what we are doing now.

G31-S2 - Yes, it's possible and you get the correct answer

G31-S1 - In the calculations? That doesn't make sense...

G31-S2 - It's not easy to imagine.

G31-S1 - It's not easy to imagine, but that's what we have been doing lately ...

G31-S2 - But (...) we should have a physical model to handle reality then, which is not only, okay, it works mathematically. But it's hard to imagine. But maybe physics is so difficult then, that there must be things we cannot imagine.

G31-S1 - Maybe.

The students here departed from an instrumental attitude where the predictive power of calculations is sufficient. However, they expressed frustration with the situation of having to accept predictions without possibility to get a mental grasp of the nature of light, indicating a realist orientation. However, student S2 also indicated that there are things we cannot describe because we cannot imagine them, in line with a Copenhagen interpretation. This indicates that students may be able to reflect on ontological questions in physics.

In total, the results show that students' conceptions span from rather naive views expecting science to prove the true nature of reality, to views that touch on ontological problems of modern physics. 


\section{Discussion}

The previous section has presented results on how upper secondary physics students' written responses and small-group discussions reveal conceptions of the nature of light and how the nature of science (NOS) is expressed through their responses. Here we discuss our findings in light of interpretations of quantum physics and the related ontological views. We also suggest implications for physics curriculum and for further research.

\subsection{Students' conceptions about the nature of light} The initial writing task "What do you think light is?" was given to students at the very beginning of the learning sequence in the ReleQuant resources. Most responses treated both the wave and the particle nature of light, with varieties saying that light is/can be both waves and particles; can behave as both waves and particles; or can be seen/described as or consists of waves and particles. These formulations resemble descriptions often found in physics textbooks. Thus, based on their previous courses in science and physics, most respondents were able to express important aspects of light even before they started their work with the ReleQuant recourses. However, there were signs of students holding an uncritical duality view, which entails that students do not reflect on the inherent contradiction between a model of light as waves and the particle model, indicating that students' understanding might be shallow. In terms of Krijtenburg-Lewerissa et al.'s (2017) summary of clusters of student understanding of waveparticle duality, many of the respondents in the present survey would be in cluster 2, a "mixed description" where a quantum physical duality perspective coexists with classical notions.

In the small-group discussions that students had after having worked with the ReleQuant resources on light, they expressed more sophisticated notions. In contrast to the written task, many students now recognized the duality paradox, and the most commonly occurring theme was light displaying either particle or wave properties depending on the situation or experiment. It is not clear from students' descriptions whether this should be interpreted as a Copenhagen stance where the act of measurement forces the system to display certain properties. It is perhaps closer to how Cini (2003) has described properties of quantum objects as "context dependent", in that the different experiments students refer to can be seen as different contexts. Although advanced explanations cannot be expected from the students at this level, some of them could probably benefit from and be motivated by going deeper into physicists' interpretations of what the duality means.

Going back to Cheong and Song's (2014) notion of different levels of the meaning of waveparticle duality, we may say that our respondents were mainly concerned with the first level of duality, namely a notion that light has both a particle property and a wave property, relating this to experimental results. The second level includes core ideas of quantum theory and "any claim of reality is not contained in the meaning of this level" (Cheong \& Song, 2014, p. 1016). This level is touched upon in later sections of the ReleQuant learning resources and is not addressed in the resources discussed here but was still mentioned by one of the respondents. However, the third level of duality is highly relevant to the present case, since Cheong and Song (2014, p. 1017) stated that "in accordance with one's philosophical position, the ultimate purpose of science could be merely predicting (or explaining) phenomena or making a reality claim". A degree of understanding of this level of duality was clearly 
present in several of our respondents' statements in the small-group discussions. Thus, whereas Cheong and Song (2014) recommended progressing from the first via the second to the third level of meaning of duality, the teaching/learning sequence we describe here tackles the first and third levels already in the beginning and addresses the second level later in the sequence.

However, there were also signs of confusion and even misunderstandings in the small-group discussions. Some groups tried to conceptually reconcile the two descriptions based on classical notions of waves and particles, in certain cases resulting in expressions of the misconception that photons are particles that move in a wave-shaped pattern or trajectory, also observed by Olsen (2002). Thus, the prompt in the form of a discussion question may for some students have resulted in articulation of a misunderstanding of wave-particle duality. A similar observation was made by McKagan et al. (2010, p. 12), who remarked that:

This particular student difficulty [the notion that photons travel along sinusoidal paths] is unlike many of the difficulties reported in introductory physics in that it is not due to prior intuition, but due to instruction. This is the only question where students sometimes do worse often much worse on the posttest than on the pretest.

One may argue that the discussion question itself ("is it possible to imagine light as both wave and particles?") begged for reconciliation of classical notions of waves and particles and expression of misconceptions. This relates to how Aspect warned that logical conflict only appears if one appeals to classical concepts (Aspect et al.1989, quoted in Greca \& Freire Jr, 2014b), which the discussion task in ReleQuant clearly does. However, based on the analysis of students' discussions, we have argued elsewhere (Bungum et al., 2018) that the way students described the idea of particles moving in waveshaped patterns in the small-group discussions signals that they most often see it as "possible to imagine" (as they are asked), but not necessarily that they hold this conception themselves. We thus argue that the teaching material supports students in making possible interpretations and conceptual dilemmas explicit rather than revealing misconceptions in students' minds.

Results from the initial writing task show that many students had a particle description of light as their starting point, even if they mentioned both models or expressed a conception of duality. This suggests that the particle model may be more intuitive whereas light as waves is more challenging to handle for students. This is corroborated in the small-group discussions where several challenges students have in comprehending light as waves are revealed. Students are familiar with waves in water in everyday life, waves on a rope or a string, and with descriptions of sound waves from science teaching. They do, however, have problems in transferring these insights to a wave model of light, and the question "what is waving?" comes up in several student discussions. This shows that light as waves is hard to envision, even if students have heard about electromagnetic waves. The challenge that students expressed shows that they are aware that light, in terms of (electromagnetic) waves, does not involve any material movement as is the case with water waves, sound and any other kinds of waves students are familiar with. Students' prior knowledge of waves thus seems to be an obstacle rather than a benefit in comprehending the wave model for light. Students also formulated questions about where a wave representing light starts, how it spreads and how something can be a wave at all when it is not anything material. 


\subsection{Students' epistemological reflections and notions about the nature of science}

Views on ontology, epistemology and the nature of science (NOS) were explicitly or implicitly present in several of the small-group discussions. The results indicate that many of the students had a relatively good basic understanding of the role a model plays in physics, in expressing an understanding of the scientific enterprise as producing models of our physical reality, and that they also accepted that several models of the same phenomenon may exist.

Like some scientists, the students showed some dissatisfaction with the situation where we have two ontologically distinct models, and several students pointed to the possibility for scientists to develop a better model in the future. Only few students expressed a conception of models as true reflections of reality. Overall, students' conceptions were hence in line with the understanding of NOS described in science education literature (Lederman \& Lederman, 2014; McComas et al., 1998). It also seems that the ReleQuant resources have been successful in making students understand how physicists may describe light in different ways, and several students referred to the video with the two physicists. How the particle and wave model are useful for describing different experimental results concerning light was also well articulated in several student discussions.

Students touched upon fundamental questions of what duality means, and whether it entails that light is particle and waves simultaneously, or if it rather means that light can change properties. This problem mirrors how Lévy-Leblond (2003) has described the wave-particle duality as at best ambiguous, since "it can be understood as meaning either that a quantum object is at once a wave and a particle, or that it is sometimes a wave and sometimes a particle" (p. 496). A related observation was made by Baily and Finkelstein (2014) who found that some students perceived switches between wave and particle descriptions as physical transitions. They emphasised the importance of attending to students' ontologies when discussing interpretations of quantum physics.

Some students also expressed a more personal need for a mental model that could help them conceptualise light. A need for mental images of what theories in physics mean is also prevalent among university physics students and working physicists (Greca \& Freire Jr, 2014b). This relates to the various functions that models have in science and in science education, as described by Gilbert (2004). He distinguished between mental models, which are an individual's personal representation of a concept or process, and scientific models developed and accepted in the community of scientists. In our material, we found that students expressed a struggle to devise mental models to make the nature of light intelligible to them personally, as well as expectations for science to produce a new and more comprehensive scientific model of light. In quantum physics, models are also mathematical tools for describing and predicting the reality we can observe, as is the case for how a mathematical model developed by Einstein led to the idea of duality. These models, however, were not introduced to the students in the present study.

The issue of how models relate to reality was only briefly addressed by some students. This indicates that many students did not consider how the meaning of the wave-particle duality should be interpreted on an ontological level. Does the duality mean that light changes properties depending on the experiment undertaken, or do different experiments expose different properties of the quantum object? Do photons "exist" independently of an observer (a realist view)? Or does it not make sense at 
all to talk about a reality beyond the theoretical models that are successful in predicting experimental results (the Copenhagen interpretation)? These questions are challenging even for physics students on more advanced university level and for researchers. The physicists may, however, perform their work with an instrumentalist interpretive approach that is in line with the views of Niels Bohr (Cordero, 2003). It is also shown that an instrumentalist approach, where students are taught how to use instrumental algorithms for calculations, is prevalent in university textbooks on quantum physics (Greca \& Freire Jr, 2014b). In contrast, many students in our study seemed to hold an underlying realist view, which is not surprising as it resembles common sense, as well as standard science practices (Bunge, 2003). Students' realist orientation is also evident in other results in research on the ReleQuant resources where students discuss the Schrödinger's cat thought experiment (Myhrehagen \& Bungum, 2016). It was found that many students saw the meaning of superposition as an expression of lack of human knowledge rather than a mixed state as an expression of a reality. This is in line with Baily and Finkelstein (2010b) who found that a significant number of students in their sample expressed a preference for realistic interpretations of quantum phenomena when instructors did not explicitly address issues of interpretation. It is then paradoxical that to the extent that a view on ontology (rather than merely an instrumental view) is made explicit in physics textbooks and instruction, it is usually only the Copenhagen interpretation that is presented (Cheong \& Song, 2014). This view has been considered to be "linked with logical positivism, a kind of anti-realism" (Cheong \& Song, 2014, p. 1015) as well as absurd, anthropomorphic and even magical (Bunge, 2003).

Our results suggest that the Copenhagen interpretation is at odds with many physics students' realist orientation. This is not mainly because students refuse the Copenhagen interpretation, but maybe more because they do not grasp what it means and do not realise how it represents an alternative to a realist view. This means that in order for students to make sense of quantum physics, teaching should pay even closer attention to what is inherent in the realist view that many students take for granted, and how this differs from the Copenhagen interpretation of quantum physics. This may also counteract tendencies that students have to hold on to conceptions from classical physics in unproductive ways when working with quantum physics (see e.g. Ayene et al., 2011; Baily \& Finkelstein, 2009; Hadzidaki, 2008).

The teaching approach in ReleQuant gives students opportunities to address the relationship between physics knowledge and reality. Results from our study indicate that when secondary school students are given access to the historical origins of quantum physical concepts and physicists' conflicting interpretations and opportunities to talk physics, they are able to reflect insightfully on fundamental philosophical questions related to quantum physics. We have shown elsewhere that the teaching approach in ReleQuant with small-group discussions can be productive in the sense that they deepen students' understanding and make them ask new questions (Bungum et al., 2018). Along similar lines, Levrini et al. (2014) observed that Italian upper secondary physics students refined their epistemological positions after a teaching intervention on optics and the nature of light where historical and philosophical aspects had a central role. The findings resonate with Krijtenburg-Lewerissa et al. (2017) recommendations for quantum physics teaching strategies, which include emphasis on interpretations along with active learning methods. 


\section{Implications and conclusions}

Science is an evolving enterprise with many open questions, leaving room for the creative explorations and interpretations of future generations of scientists. Many have argued that the history and philosophy of science should be part of science teaching (see e.g. Garritz, 2013; Kragh, 1992), and Greca and Freire Jr (2014b) emphasized that the teaching of quantum physics at the university level, maybe more than other subject areas in physics, must be informed by the history and philosophy of science. Based on our results, we will argue that this is also the case for pre-university physics teaching, as students are already on this stage encultured into a physics culture dominated by classical physics and its underlying epistemology and ontology.

Greca and Freire Jr (2014a) have noted that few studies on the teaching of quantum physics refer to different possible interpretations on an ontological level. Our results from the present study indicate that students tend to implicitly hold a realist view and have problems in seeing alternatives. Similar results have been found in the study by Baily and Finkelstein (2009) on undergraduate university students in physics. We therefore suggest that the ontological foundation for the Copenhagen interpretation and how it differs from a realist view should be made explicit for physics students already at the secondary level. Students should be made aware that theories in quantum mechanics involve mathematical models that are successful in predicting outcomes of experiments, but that the interpretation of these theories is still subject to debate.

In order to offer students a realist description of light avoiding the challenges with the ontological basis of the Copenhagen school, Bunge (2003) and other authors have argued that physics teaching should avoid the notion of wave-particle duality. They suggested instead to introduce a concept of "real" quantum objects (quantons) distinct from waves as well as particles in a classical sense. However, we fear that this strategy in teaching quantum physics on a basic level may risk introducing yet another new, abstract concept that is not easily accessible and that challenges students' need to build a mental model of reality. Moreover, Lautesse et al. (2015) pointed out that among French school physics textbooks, even the ones that adopted the quanton concept resorted to wave and particle concepts in order to describe it, and the authors suggested that the term may cause more problems than those it resolves for upper secondary students. Thus, getting rid of the wave and particle concepts when describing light and other quantum phenomena does not seem feasible or even possible. The concept of context dependence for quantum objects as described by Cini (2003) may, however, be accessible for students, as our results suggest that many students' descriptions of quantum phenomena may be in line with this idea.

Students in our study expressed challenges in envisioning what the wave model for light entails. The results suggest that light as waves needs to be further discussed with physics students, in particular since the wave properties of light are so essential in explaining phenomena in more advanced physics that students may encounter at a later stage of their education. Even if it has been suggested that the teaching of quantum physics should avoid classical pictures (Ayene et al., 2011; Baily \& Finkelstein, 2009; Hadzidaki, 2008), descriptions of light as "waves" will inevitably produce associations of waves in a classical sense for students. Our results show that these are unproductive as long as students are not given more constructive pictures of what "waves" means with regard to light. Connection to matter 
waves and the key characteristic of quantum objects as blunt rather than sharp as described by Bunge (2003) may contribute to creating constructive images in this regard, and should be an area for further research and development.

Our findings extend the body of research addressing pre-university students' conceptions about quantum physics, notably by addressing conceptions of light in the context of the ongoing discussion of interpretations of quantum physics and how these can be included in physics instruction. We have documented that upper secondary physics students are able to grasp and discuss central dilemmas in the interpretation of wave-particle duality. We have also documented conceptual challenges students encounter, and how recognising different interpretations of quantum physics is a challenge for students. Like several other authors (Baily \& Finkelstein, 2010b; Cheong \& Song, 2014; Greca \& Freire Jr, 2014a), we recommend that students should be made aware that there is still no consensus on basic questions regarding interpretation of quantum physics. A historical perspective on quantum physics may represent a suitable entry point for students to explore the development and interpretation of quantum physical concepts.

A physics subject less focused on "correct explanations" and more open to philosophical discussions may contribute to attracting a larger and more diverse group of learners to higher education in physics, since the traditional discipline culture of physics is known to attract learners with a traditional, narrowly content-centred interest in the subject (Angell, Guttersrud, Henriksen, \& Isnes, 2004; Bøe \& Henriksen, 2013; Carlone, 2004). The physics students in our investigation are encultured into a traditional physics disciplinary culture which tends to focus on established content knowledge rather than unresolved questions and historical-philosophical aspects (Bøe et al., in press). Approaches focusing on collaborative learning, discussion and philosophical questions may broaden this discipline culture and make it more inclusive. Thus, our study is an example of how historical-philosophical aspects and new teaching approaches may contribute to renewing physics teaching and learning, supporting students in making sense of quantum physics and familiarizing them with physics as an intellectual and creative human endeavour in constant development.

\section{Acknowledgements:}

The authors would like to thank participating students and teachers and the ReleQuant project group for their contributions. This work was supported by a grant from the Research Council of Norway (project no 246723) and by the Olav Thon Foundation. 


\section{References}

Abd-El-Khalick, F. (2012). Examining the sources for our understandings about science: Enduring conflations and critical issues in research on nature of science in science education. International Journal of Science Education, 34(3), 353-374.

Anderson, T., \& Shattuck, J. (2012). Design-based research: A decade of progress in education research? Educational researcher, 41(1), 16-25.

Angell, C., Guttersrud, Ø., Henriksen, E. K., \& Isnes, A. (2004). Physics: Frightful, but fun. Pupils' and teachers' views of physics and physics teaching. Science Education, 88(5), 683-706.

Arons, A., \& Peppard, M. (1965). Einstein's Proposal of the Photon Concept-a Translation of the Annalen der Physik Paper of 1905. American Journal of Physics, 33(5), 367-374.

Ayene, M., Kriek, J., \& Damtie, B. (2011). Wave-particle duality and uncertainty principle: Phenomenographic categories of description of tertiary physics students' depictions. Physical Review Special Topics-Physics Education Research, 7(2), 020113.

Baily, C., \& Finkelstein, N. D. (2009). Development of quantum perspectives in modern physics. Physical Review Special Topics-Physics Education Research, 5(1), 010106.

Baily, C., \& Finkelstein, N. D. (2010a). Refined characterization of student perspectives on quantum physics. Physical Review Special Topics-Physics Education Research, 6(2), 020113.

Baily, C., \& Finkelstein, N. D. (2010b). Teaching and understanding of quantum interpretations in modern physics courses. Physical Review Special Topics-Physics Education Research, 6(1), 010101.

Baily, C., \& Finkelstein, N. D. (2014). Ontological Flexibility and the Learning of Quantum Mechanics. arXiv preprint arXiv:1409.8499.

Bohr, N. (1928). The Quantum Postulate and the Recent Development of Atomic Theory. Nature, 121, 580-590.

Bohr, N. (1961). Atomic theory and the description of nature. Cambridge: Cambridge University Press.

Braun, V., \& Clarke, V. (2006). Using thematic analysis in psychology. Qualitative Research in Psychology, $3(2), 77-101$.

Bunge, M. (2003). Twenty-five centuries of quantum physics: From Pythagoras to us, and from subjectivism to realism. Science \& Education, 12(5), 445-466.

Bunge, M. (2012). Does Quantum Physics Refute Realism, Materialism and Determinism? Science \& Education, 21(10), 1601-1610. doi:10.1007/s11191-011-9410-z

Bungum, B., Bøe, M. V., \& Henriksen, E. K. (2018). How small-group discussions may enhance students' understanding in quantum physics. Unpublished manuscript. NTNU. Trondheim.

Bungum, B., Henriksen, E. K., Angell, C., Tellefsen, C. W., \& Bøe, M. V. (2015). ReleQuant-Improving teaching and learning in quantum physics through educational design research. Nordic Studies in Science Education, 11(2), 153-168.

Bøe, M. V., \& Henriksen, E. K. (2013). Love it or leave it: Norwegian students' motivations and expectations for postcompulsory physics. Science Education, 97(4), 550-573.

Bøe, M. V., Henriksen, E. K., \& Angell, C. (in press). Actual vs. implied physics students: How students from traditional physics classrooms related to an innovative approach to quantum physics. Science Education. doi:10.1002/sce.21339

Camilleri, K. (2009). Constructing the myth of the Copenhagen interpretation. Perspectives on Science, 17(1), 26-57.

Carlone, H. B. (2004). The cultural production of science in reform - based physics: Girls' access, participation, and resistance. Journal of research in science teaching, 41(4), 392-414. 
Cheng, M.-F., \& Lin, J.-L. (2015). Investigating the relationship between students' views of scientific models and their development of models. International Journal of Science Education, 37(15), 2453-2475.

Cheong, Y. W., \& Song, J. (2014). Different levels of the meaning of wave-particle duality and a suspensive perspective on the interpretation of quantum theory. Science \& Education, 23(5), 1011-1030.

Cini, M. (2003). How Real is the Quantum World? Science \& Education, 12(5), 531-540.

Cordero, A. (2003). Understanding quantum physics. Science \& Education, 12(5), 503-511.

Einstein, A. (1989). The Collected Papers of Albert Einstein. Vol. 2: The Swiss Years: Writings, 1900-1909, edited by J. Stachel Princeton. In: NJ: Princeton University Press.

Garritz, A. (2013). Teaching the philosophical interpretations of quantum mechanics and quantum chemistry through controversies. Science \& Education, 22(7), 1787-1807.

Gilbert, J. K. (2004). Models and modelling: Routes to more authentic science education. International Journal of Science and Mathematics Education, 2(2), 115-130.

Gingras, Y. (2015). The creative power of formal analogies in physics: the case of Albert Einstein. Science \& Education, 24(5-6), 529-541.

Gjerland, M. (2015). Elevers oppfatning om lys og bølge/partikkel-dualismen (Students' understanding of light/wave dualism). (Master's thesis), NTNU, Trondheim.

Greca, I. M., \& Freire Jr, O. (2014a). Meeting the challenge: quantum physics in introductory physics courses. In International Handbook of Research in History, Philosophy and Science Teaching (pp. 183-209): Springer.

Greca, I. M., \& Freire Jr, O. (2014b). Teaching introductory quantum physics and chemistry: caveats from the history of science and science teaching to the training of modern chemists. Chem. Educ. Res. Pract., 15, 286-296.

Greca, I. M., \& Freire, O. (2003). Does an emphasis on the concept of quantum states enhance students' understanding of quantum mechanics? Science \& Education, 12(5), 541-557.

Hadzidaki, P. (2008). Quantum mechanics' and 'scientific explanation'an explanatory strategy aiming at providing 'understanding. Science \& Education, 17(1), 49-73.

Held, C. (1994). The meaning of complementarity. Studies in History and Philosophy of Science Part A, 25(6), 871-893.

Henriksen, E. K., \& Angell, C. (2010). The role of 'talking physics' in an undergraduate physics class using an electronic audience response system. Physics Education, 45(3), 278.

Henriksen, E. K., Bungum, B., Angell, C., Tellefsen, C. W., Frågåt, T., \& Bøe, M. V. (2014). Relativity, quantum physics and philosophy in the upper secondary curriculum: challenges, opportunities and proposed approaches. Physics Education, 49(6), 678.

Hubber, P. (2006). Year 12 students' mental models of the nature of light. Research in Science Education, 36(4), 419-439.

Ireson, G. (1999). A multivariate analysis of undergraduate physics students' conceptions of quantum phenomena. European Journal of Physics, 20(3), 193.

Ireson, G. (2000). The quantum understanding of pre-university physics students. Physics Education, 35(1), 15.

Karakostas, V., \& Hadzidaki, P. (2005). Realism vs. constructivism in contemporary physics: the impact of the debate on the understanding of quantum theory and its instructional process. Science \& Education, 14(7), 607-629.

Kragh, H. (1992). A sense of history: history of science and the teaching of introductory quantum theory. Science \& Education, 1(4), 349-363.

Kragh, H., \& Pedersen, S. A. (1992). Naturvidenskabens teori (The philosophy of science). Copenhagen: Nyt Nordisk Forlag Arnold Busck. 
Krijtenburg-Lewerissa, K., Pol, H. J., Brinkman, A., \& van Joolingen, W. (2017). Insights into teaching quantum mechanics in secondary and lower undergraduate education. Physical review physics education research, 13(1), 010109.

Lautesse, P., Valls, A. V., Ferlin, F., Héraud, J.-L., \& Chabot, H. (2015). Teaching quantum physics in upper secondary school in France. Science \& Education, 24(7-8), 937-955.

Lederman, N. G., Abd-El-Khalick, F., Bell, R. L., \& Schwartz, R. S. (2002). Views of nature of science questionnaire: Toward valid and meaningful assessment of learners' conceptions of nature of science. Journal of research in science teaching, 39(6), 497-521.

Lederman, N. G., \& Lederman, J. S. (2014). Research on teaching and learning of nature of science. In N. G. Lederman \& S. K. Abell (Eds.), Handbook of research on science education. Abingdon, England: Routledge.

Levrini, O., Bertozzi, E., Gagliardi, M., Tomasini, N. G., Pecori, B., Tasquier, G., \& Galili, I. (2014). Meeting the Discipline-Culture Framework of Physics Knowledge: A Teaching Experience in Italian Secondary School. Science \& Education, 23(9), 1701-1731.

Lévy-Leblond, J. M. (2003). On the Nature of Quantons. Science \& Education, 12, 495-502.

Mannila, K., Koponen, I. T., \& Niskanen, J. A. (2001). Building a picture of students' conceptions of waveand particle-like properties of quantum entities. European Journal of Physics, 23(1), 45-53.

McComas, W. F., Almazroa, H., \& Clough, M. P. (1998). The nature of science in science education: An introduction. Science \& Education, 7(6), 511-532.

McKagan, S., Perkins, K., \& Wieman, C. (2010). Design and validation of the quantum mechanics conceptual survey. Physical Review Special Topics-Physics Education Research, 6(2), 020121.

Myhrehagen, V. H., \& Bungum, B. (2016). From the cat's point of view': upper secondary physics students' reflections on Schrödinger's thought experiment. Physics Education, 51(5), 055009.

NDET. (2006). Physics - programme subject in programmes for specialization in general studies. Retrieved from https://www.udir.no/kl06/FYS1-01?lplang=http://data.udir.no/kl06/eng

Newton, I. (1952). Opticks, or, a treatise of the reflections, refractions, inflections \& colours of light: Courier Corporation.

NGSS. (2013). Next generation science standards: For states, by states. In. Washington, DC: National Academies Press.

Olsen, R. V. (2002). Introducing quantum mechanics in the upper secondary school: a study in Norway. International Journal of Science Education, 24(6), 565-574.

Planck, M. (1900). On the theory of the energy distribution law of the normal spectrum. In H. Kangro (Ed.), Planck' origional papers in quantum physics (pp. 38 - 45). London: Taylor and Francis.

Renstrøm, R. (2011). Kvantefysikkens utvikling - i fysikklærebøker, vitenkapshistorien og undervisning [The development of quantum physics - in physics textbooks, in the history of science, and in the classroom]. (Ph.D. thesis), University of Oslo.

Vervoort, L., \& Gingras, Y. (2015). Macroscopic Oil Droplets Mimicking Quantum Behaviour: How Far Can We Push an Analogy? International Studies in the Philosophy of Science, 29(3), 271-294.

Vygotsky, L. (1978). Mind in society (M. Cole, Ed.). Cambridge, Massachusetts: Harvard.

Young, T. (1804). The Bakerian lecture: Experiments and calculations relative to physical optics. Philosophical transactions of the Royal Society of London, 94, 1-16. 